\title{
Análisis de concentraciones plasmáticas de voriconazol y su perfil de seguridad en pacientes oncológicos pediátricos
}

\author{
Felipe Silva, Daniel Navea, Carolina Salas, Juan P. Torres, Paula Catalán y Jorge Morales
}

\section{Analysis of voriconazole serum concentrations and safety profile in pediatric oncology patients}

Background: Voriconazole (VCZ) serum drug levels (SDL) vary widely and are associated with increased mortality when they are below the therapeutic range for invasive aspergillosis (IA). Aim: To describe VCZ SDL in oncology pediatric patients in order to reach adequate concentrations for prophylaxis $(\geq 0.5 \mathrm{mg} / \mathrm{L})$ and treatment $(\geq 1.0$ y $2.0 \mathrm{mg} / \mathrm{L})$ for IA and their relationship with toxicity. Patients and Methods: Retrospective analysis of VCZ SDL and toxicities recorded in oncology pediatric patients between February 2013 and November 2014. The daily dosage and SDLs were analyzed according to administration route: intravenous (IV) and oral (PO), type of therapy (prophylaxis and treatment) and patient age ( $<12 \mathrm{y} \geq 12$ years old). Results: 112 through levels from 26 patients were analyzed and the average age was 9.3 years-old. The SDL obtained from the IV route were $43.7 \%$. There were more SDL $\geq 0.5 \mathrm{mg} / \mathrm{L}$ and $\geq 1.0 \mathrm{mg} / \mathrm{L}$ with the IV route than the PO route $(\mathrm{p}<0.05)$. Patients younger than 12-years-old received a higher dosage than those $\geq 12$ years old (median 18.6 and $9.2 \mathrm{mg} / \mathrm{kg} / \mathrm{d}$, respectively, $\mathrm{p}<0.05$ ). To reach SDL $\geq 0,5 \mathrm{mg} / \mathrm{L}$ with the PO route, a dosage of $200 \mathrm{mg}$ every 12 hours showed the best results for all patients (80-100\% SDL $\geq 0.5 \mathrm{mg} / \mathrm{L})$. With an IV dosage between 14 and $20 \mathrm{mg} / \mathrm{kg} /$ day in patients $\geq 12$-yearsold, $80 \%$ of the SDL were $\geq 1 \mathrm{mg} / \mathrm{L}$ and $\geq 2 \mathrm{mg} / \mathrm{L}$. In patients younger than 12-year-old, dosages between $8-30 \mathrm{mg} /$ $\mathrm{kg}$ /day showed similar results (50-63\% of SDL $\geq 1 \mathrm{mg} / \mathrm{L}$ and $36-40 \%$ of SDL $\geq 2 \mathrm{mg} / \mathrm{L}$ ). Eight patients (30.8\%) presented an adverse drug reaction and no relationship with the SDL was found. Conclusion:A VCZ standard dosage of $200 \mathrm{mg}$ every 12 hours PO showed the best results for IA prophylaxis in all patients. Patients younger than 12-years-old would require higher dosages than the doses used in this study to attain adequate SDL for IA treatment. No relation with SDL and adverse reactions was found.

Key words: Voriconazole, serum concentration, pharmacokinetics, adverse drug reactions.

Palabras clave: Voriconazol, concentraciones plasmáticas, farmacocinética, reacciones adversas.

\section{Introducción}

$\mathrm{V}$ oriconazol (VCZ) es un antifúngico triazólico de amplio espectro disponible, tanto en presentación vía oral (vo) como intravenosa (iv). Actúa inhibiendo la enzima 14- $\alpha$-esterol desmetilasa, interrumpiendo la síntesis de la membrana fúngica ${ }^{1}$ y posee acción contra especies de Aspergillus, Candida, Fusarium y Scedosporium. Es recomendado como fármaco de primera línea en el tratamiento de aspergilosis invasora (AI) en pacientes inmunocomprometidos ${ }^{2}$.

La profilaxis con antifúngicos, tanto en pacientes con factores de riesgo de adquirir una AI (profilaxis primaria) así como pacientes con antecedentes que AI que cursen períodos de inmunosupresión (profilaxis secundaria), es necesaria, ya que de no hacerlo, aumenta de manera significativa el riesgo de recaída de la enfermedad fúngica y de mortalidad ${ }^{3}$. Aunque posaconazol es el fármaco con registro de la U.S Food and Drug Administration (FDA) para profilaxis de $\mathrm{AI}^{4}$, hay evidencias para el uso exitoso de $\mathrm{VCZ}$ en pacientes pediátricos, tanto en profilaxis primarias como secundarias ${ }^{5-7}$.

Farmacocinética: su absorción disminuye en presencia de alimentos, donde su concentración plasmática máxima (Cmáx) y área bajo la curva (ABC) se pueden reducir hasta en 34 y $24 \%$, respectivamente ${ }^{8,9}$. Los pacientes pediátricos presentan además una menor absorción oral $(44,6-65 \%)^{10,11}$ en comparación a pacientes adultos (96\%). Voriconazol es metabolizado principalmente en el hígado por las enzimas CYP2C19, CYP3A4, y en menor grado por $\mathrm{CYP} 2 \mathrm{C}^{12}$. Otra característica de los pacientes pediátricos es que presentan un aumento del metabolismo pre-sistémico de este fármaco (efecto de primer paso hepático), lo que también contribuye a la obtención de concentraciones plasmáticas (CPs) más bajas ${ }^{10}$. Su distribución es bi-compartimental y su eliminación sigue el modelo de Michaelis-Menten, es decir, tiene un metabolismo saturable que provoca una farmacocinética no lineal. Por esto, con un aumento de dosis, la CP puede aumentar de manera desproporcional, lo que complica aún
Hospital Dr. Luis Calvo Mackenna, Santiago, Chile. Unidad de Infectología (JPY) Unidad de Trasplante de Precursores Hematopoyéticos (PC).

Unidad de Farmacia Clínica (FS, JM). Unidad de Laboratorio, sección Farmacocinética (DN, CS). Universidad de Chile. Facultad de Medicina, Departamento de Pediatría y Cirugía Infantil Oriente (JPT).

Los autores declaran no tener conflictos de intereses.

Este trabajo no contó con fuentes de financiamiento.

Recibido: 5 de agosto de 2015 Aceptado: 22 de febrero de 2016

Correspondencia a: Felipe Silva Marambio felipe.silva.marambio@gmail.com 
más la dosificación de este fármaco. Es importante señalar que la presencia de polimorfismos en la secuencia genética que codifica la enzima CYP2C19, genera enzimas con diferente capacidad metabólica, aumentando o disminuyendo las CPs de VCZ. La variabilidad de CPs, tanto intra-paciente así como inter-paciente, ha demostrado ser alta $^{13,14}$ y los factores que influyen en esta variabilidad son: polimorfismo de la enzima CYP2C19, edad, sexo, interacciones farmacológicas y enfermedad hepática?

\section{Monitorización de concentraciones plasmáticas}

El control de CPs de VCZ es recomendado en pacientes adultos y pediátricos por la Infectious Diseases Society of America (IDSA) (recomendación B-III) ${ }^{4}$. Una concentración baja de $\mathrm{VCZ}$ al inicio del tratamiento de una infección fúngica invasora (IFI) es considerada como un factor de riesgo para el aumento de mortalidad en estos pacientes ${ }^{15}$. Un estudio observacional en adultos encontró que una $\mathrm{CP}$ basal de $1 \mathrm{mg} / \mathrm{L}$ estuvo relacionada con $70 \%$ de éxito terapéutico ${ }^{16}$. Por otra parte, en pediatría, en un estudio retrospectivo de 46 pacientes, CPs $<1 \mathrm{mg} / \mathrm{L}$ se asociaron con un incremento 2,6 veces en el riesgo de mortalidad $^{17}$.

La elevada variabilidad de las CPs y la falta de definiciones claras para su dosificación en pediatría hacen que la obtención de CPs terapéuticas, tanto con su administración intravenosa (iv) así como vía oral (vo), sea un desafío.

El objetivo primario de este estudio es describir las CPs de VCZ obtenidas de pacientes pediátricos que recibieron tratamiento iv o vo, y compararlas con la dosis recibida. Nuestro objetivo secundario es analizar la existencia de alguna asociación entre CPs y reacciones adversas.

\section{Materiales y Métodos}

\section{Diseño del estudio y tipo de pacientes}

Estudio observacional, retrospectivo, realizado en el Hospital Pediátrico Dr. Luis Calvo Mackenna (Santiago, Chile), institución estatal de alta complejidad que cuenta con 190 camas, entre las que se incluyen las unidades clínicas de Oncología y de Trasplante de Precursores Hematopoyéticos. Se revisaron en los registros de la Unidad de Farmacia Clínica las CPs valle de VCZ obtenidas en pacientes inmunocomprometidos de 2 a 18 años, durante el período comprendido entre febrero de 2013 y noviembre de 2014 .

\section{Criterios de inclusión y exclusión}

Se incluyeron pacientes oncológicos y aquellos receptores de trasplante de precursores hematopoyéticos. Todos recibieron VCZ $\left(\right.$ VFEND $^{\circledR}$, Pfizer, Inc) en su presentación iv (ampollas de $200 \mathrm{mg}$ ) y/o vo (comprimidos de libera- ción convencional de $200 \mathrm{mg}$ ), de acuerdo al protocolo establecido en el hospital (el criterio para indicar la vía de administración se detalla más adelante). Se incluyeron todos aquellos pacientes con mediciones de CPs valle, independiente del tipo de infección fúngica y tipo de terapia (tratamiento o profilaxis). Se consideraron $C P S$ valle aquellas obtenidas dentro de 30 minutos previos a la siguiente dosis, tras al menos cinco días de iniciarse el tratamiento con VCZ, requisitos que fueron monitorizados por la unidad de farmacia clínica. Este estudio no requirió de consentimiento/asentimiento informado debido a que la monitorización de CPs es una práctica protocolizada en la institución; no se utilizaron datos personales de los pacientes para la publicación del estudio.

Se excluyeron los pacientes con: hipersensibilidad previa a antifúngicos azólicos, control de $\mathrm{CP}$ que fuera efectuado antes de cinco días de iniciado el tratamiento, función hepática alterada previo inicio al tratamiento (enzimas alanina aminotransferasa (ALT) y/o aspartato aminotransferasa $($ AST $)>5$ veces valor normal, o bilirrubinemia total $>2,5$ valor normal (según valores normales por rango de edad), depuración de creatinina $<50 \mathrm{ml} /$ $\min / 1,73 \mathrm{~m}^{2}$ para administración iv, administración vo con ingesta de alimentos con menos de $1 \mathrm{~h}$ antes o post administración del fármaco (incluye nutrición enteral), tratamiento concomitante con fármacos inductores $\mathrm{o}$ inhibidores fuertes del CYP3450 y concentraciones plasmáticas pico obtenidas una hora post administración del fármaco.

Dosificación: se utilizó la formulación iv en pacientes con IFI o sospecha de ésta y en aquellos con indicación de profilaxis pero presentaban mala tolerancia oral o condiciones que disminuyen la absorción del fármaco (mucositis, diarrea, vómitos o enfermedad de injerto versus hospedero-EICH digestivo). La dosis inicial vía iv fue de $14 \mathrm{mg} / \mathrm{kg} /$ día fraccionado cada $12 \mathrm{~h} \mathrm{(7} \mathrm{mg/kg/}$ dosis) ajustada a la presentación de la ampolla (200 mg) con un máximo de $600 \mathrm{mg}$ al día. La formulación oral se utilizó en pacientes ingresados al hospital, que ya estuviesen recibiendo el fármaco como profilaxis para $\mathrm{AI}$ y en pacientes en tratamiento iv con al menos dos CPs valle consecutivas en rango óptimo, mejoría clínica y buena tolerancia oral, que permitiese realizar el cambio a vo. La dosis oral fue de $200 \mathrm{mg}$ cada 12 o 24 h, según fuese la situación clínica y el tipo de paciente ${ }^{10,17}$. El ajuste de dosis fue individualizado en cada paciente para alcanzar $\mathrm{CPs} \geq 1-2 \mathrm{mg} / \mathrm{L}$ para tratamiento de $\mathrm{AI} y \geq 0,5 \mathrm{mg} / \mathrm{L}$ para pacientes que requirieran profilaxis contra $\mathrm{AI}^{18,19}$.

Las solicitudes de medición de CPs, ajustes y/o mantención de dosis, junto con la prevención o manejo de interacciones medicamentosas y monitorización de reacciones adversas fueron realizadas por la unidad de farmacia clínica (JM y FS). Los datos registrados por las mismas personas fueron: nombre del paciente, edad, sexo, 
diagnóstico de base, servicio clínico, vía de administración (iv o vo), frecuencia de administración (cada 24, 12 u $8 \mathrm{~h}$ ), dosis total diaria (mg/día), dosis por kilógramo de peso $(\mathrm{mg} / \mathrm{kg} /$ día $)$ y CP $(\mathrm{mg} / \mathrm{L})$.

\section{Farmacovigilancia}

Se consultaron en los registros de la Unidad de Farmacia Clínica las reacciones adversas a medicamentos (RAM) ocurridas en los pacientes tratados con VCZ. Éstas fueron clasificadas y graduadas de acuerdo a la Common Terminology Criteria for Adverse Events, versión $4.03^{20}$. La causalidad de las RAM fue determinada utilizando el algoritmo de Naranjo ${ }^{21}$. Se realizó una búsqueda de exámenes de función hepática en la base de datos de exámenes de laboratorio del hospital $\left(\operatorname{SysLAB}{ }^{\circledR}\right)$. Se incluyeron exámenes intra-tratamiento y hasta $48 \mathrm{~h}$ post control de la CP. Las pruebas registradas fueron: ALT, AST, bilirrubinemia directa y total, concentración plasmática de fosfatasa alcalina, gamma glutamil transpeptidasa (GGT), amilasa y lipasa.

\section{Obtención de muestra y medición de CPs}

Se controló la CP valle de acuerdo a la descripción anterior. Para cada muestra se extrajo $2 \mathrm{ml}$ de sangre periférica, la que se recolectó en tubos con EDTA y luego se centrifugó a 400 x g para separar el plasma, transfiriéndolo inmediatamente a un tubo Eppendorf. El plasma fue almacenado hasta su análisis a $-20^{\circ} \mathrm{C}$. La muestra fue analizada mediante cromatografía líquida de alta resolución, con extracción líquido-líquido y detección mediante detector de arreglo de diodos ${ }^{22}$, en la sección de Farmacocinética de la Unidad de Laboratorios de nuestro hospital.

\section{Análisis estadístico}

Se utilizaron pruebas estadísticas no paramétricas ya que se asumió una distribución no normal de la muestra. Para el análisis de dos muestras independientes se utilizó la prueba de Mann-Whitney. Para el análisis de datos categóricos se utilizó la prueba de $\chi^{2}$, mientras que la prueba de Fisher fue utilizada cuando la muestra analizada fue muy pequeña $(\leq 5)$. Se aceptó un error alfa de $5 \%$ y el cálculo de dichas pruebas fue realizado utilizando el paquete estadístico VassarStats ${ }^{\circledR 23}$.

\section{Resultados}

Se revisaron las CPs de VCZ obtenidas en el período antes señalado. La información de las CPs y pacientes incluidos se detallan en la Tabla 1. Se incluyó un total de 112 CPs correspondientes a 26 pacientes, de los cuales ocho $(30,8 \%)$ fueron $\geq 12$ años. Diez y seis pacientes $(61,5 \%)$ tenían patología oncológica y no habían sido sometidos a trasplante de precursores hematopoyéticos.

El análisis comparativo de CPs valle obtenidas con la administración iv y vo se muestra en la Tabla 2. El número de CPs (\%) correspondiente a administración iv y vo fue de $49(43,7 \%)$ y $63(56,3 \%)$, respectivamente. Si bien los grupos fueron comparables en cuanto a edad, peso y dosis en $\mathrm{mg} / \mathrm{kg}$ recibida, hubo una diferencia significativa para la dosis total diaria, en la que pacientes con administración iv recibieron, en promedio, una dosis mayor.

En la Figura 1 se muestra el porcentaje de CPs que alcanzaron los valores recomendados. Sólo $74 \%$ de todas las CPs fueron $\geq 0,5 \mathrm{mg} / \mathrm{L}$. Sin embargo, la cantidad de CPs sobre este rango fue significativamente mayor con la administración iv en comparación a la vo ( 84 vs $62 \%$,

\begin{tabular}{|ll|}
\hline Tabla 1. Características demográficas de los pacientes \\
\hline Parámetro & Valor \\
\hline Número de pacientes & 26 \\
Sexo masculino $n(\%)$ & $15(57,7)$ \\
\hline Edad: mediana [rango] & $9[2-18]$ \\
\hline Diagnóstico de base $\mathbf{n}(\%)$ & \\
\hline Trasplante de precursores hematopoyéticos & $10(38,5)$ \\
Tumores sólidos & $7(26,9)$ \\
Leucemia linfoblástica aguda & $6(23,1)$ \\
Aplasia medular & $2(7,7)$ \\
Leucemia mieloide aguda & $1(3,8)$ \\
\hline
\end{tabular}

Tabla 2. Comparación de datos demográficos y dosis recibidas entre concentraciones plasmáticas obtenidas con la formulación intravenosa y vía oral

\begin{tabular}{|c|c|c|c|c|}
\hline Parámetro & Total & Intravenosa & Vía oral & $p$ \\
\hline CPs valle $\mathrm{n}(\%)$ & $112(100)$ & $49(43,7)$ & $63(56,3)$ & \\
\hline \multicolumn{5}{|l|}{ Edad (años) } \\
\hline Mediana [rango] & $9[2-18]$ & $6[2-18]$ & $7[3-18]$ & $0,8337^{1}$ \\
\hline \multicolumn{5}{|l|}{ Peso (kg) } \\
\hline Mediana [rango] & $28[10-67]$ & 28,6 [10-67] & 23 [17-60] & $0,1802^{1}$ \\
\hline \multicolumn{5}{|l|}{ Dosis diaria (mg) } \\
\hline Mediana [rango] & $400[140-600]$ & $400[140-600]$ & $400[200-600]$ & $0,0414^{1 *}$ \\
\hline \multicolumn{5}{|c|}{ Dosis por peso $(\mathrm{mg} / \mathrm{kg})$} \\
\hline Mediana [rango] & $15[3,3-30,0]$ & $14[6,0-30,0]$ & $16,6[3,3-25,0]$ & $0,5552^{1}$ \\
\hline
\end{tabular}




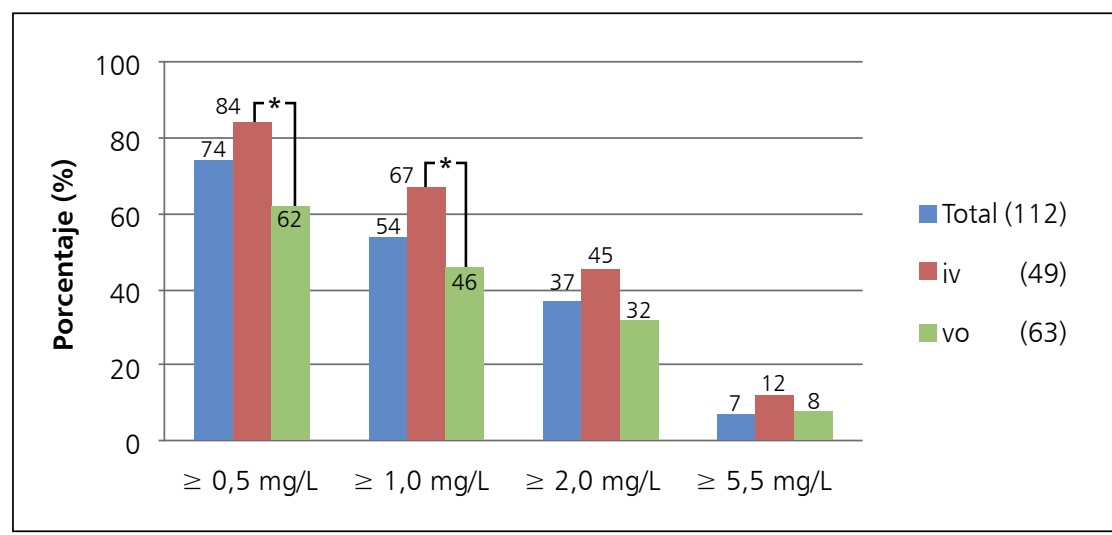

Figura 1. Comparación de CPs de voriconazol obtenidas con administración iv y vo. *Diferencia estadística significativa $(p<0,05)$, prueba de $\chi^{2}$.

Tabla 3. Comparación de concentraciones plasmáticas de voriconazol entre pacientes $<12$ años $y \geq 12$ años

\begin{tabular}{|c|c|c|c|}
\hline Parámetro & $\begin{array}{l}\text { Pacientes } \\
<12 \text { años }\end{array}$ & $\begin{array}{l}\text { Pacientes } \\
\geq 12 \text { años }\end{array}$ & p \\
\hline Pacientes n (\%) & $18(69,2)$ & $8(30,8)$ & \\
\hline Determinación de CP n (\%) & $74(66,1)$ & $38(33,9)$ & \\
\hline CPs con vía iv: $\mathrm{n}(\%)$ & $32(65,3)$ & $17(34,7)$ & \\
\hline CPs con vo: $\mathrm{n}(\%)$ & $42(66,7)$ & $21(33,3)$ & \\
\hline $\mathrm{CP}$ (mg/L): mediana [rango] & $0,94[0,1-15,5]$ & $1,75[0,10-18,0]$ & $0,1676^{1}$ \\
\hline Dosis (mg/kg/día): mediana [rango] & $18,6[5,3-30,0]$ & $9,2[3,3-24,0]$ & $0,0414^{1 *}$ \\
\hline $\mathrm{CPs} \geq 0,5 \mathrm{mg} / \mathrm{L} \mathrm{n}(\%)$ & $53(71,6)$ & $27(71,1)$ & $0,9203^{2}$ \\
\hline $\mathrm{CPs} \geq 1,0 \mathrm{mg} / \mathrm{L} \mathrm{n}(\%)$ & $35(47,3)$ & $22(57,9)$ & $0,1715^{2}$ \\
\hline $\mathrm{CPs} \geq 2,0 \mathrm{mg} / \mathrm{L} \mathrm{n}(\%)$ & $23(31,1)$ & $15(39,5)$ & $0,2713^{2}$ \\
\hline $\mathrm{CPs} \geq 5,5 \mathrm{mg} / \mathrm{Ln}(\%)$ & $5(6,8)$ & $2(5,3)$ & $0,8875^{2}$ \\
\hline
\end{tabular}

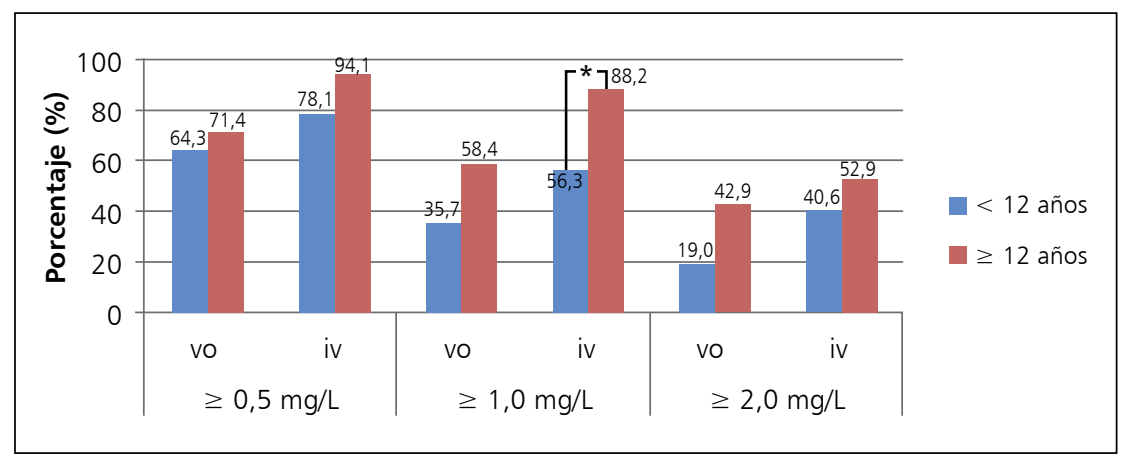

Figura 2. CPs de voriconazol en pacientes $<12$ años $y \geq 12$ años con administración vo e iv. *Diferencia estadística significativa, test de Fisher $p<0,05$. $\mathrm{p}<0,05)$. Respecto a las CPs efectivas para tratamiento de AI, una cantidad significativamente mayor de CPs fueron $\geq 1,0 \mathrm{mg} / \mathrm{L}$ con la administración iv en comparación a la administración vo ( 67 vs $46 \%, \mathrm{p}<0,05)$. Si bien hubo una mayor cantidad de $\mathrm{CPs} \geq 2,0 \mathrm{mg} / \mathrm{L}$ con la administración iv, esta diferencia no fue significativa (p: 0,219).

Sólo 7\% de todas las CPs fueron consideradas tóxicas ( $\geq 5,5 \mathrm{mg} / \mathrm{L}$ ), las que se presentaron con ambas vías de administración. Los efectos tóxicos clínicos y de laboratorio se detallan más adelante.

\section{Análisis por edad}

$\mathrm{El}$ análisis por edad para pacientes $<12$ años $\mathrm{y} \geq 12$ años se muestra en la Tabla 3. Sesenta y nueve por ciento de los pacientes tenían menos de 12 años y $66 \%$ de las determinaciones de CPs correspondieron a este grupo. Si bien se observan CPs más elevadas en el grupo $\geq 12$ años, esta diferencia no fue significativa. Sin embargo, los pacientes bajo 12 años de edad recibieron una dosis significativamente mayor en $\mathrm{mg} / \mathrm{kg} /$ día $(\mathrm{p}<0,05)$. Sin considerar la vía de administración, no se observó diferencia significativa alguna entre ambos grupos para el porcentaje de CPs sobre los valores de análisis estudiados.

En la Figura 2 se muestra la comparación de CPs obtenidas entre la administración iv y vo. En general, los porcentajes de CPs sobre los valores estudiados fueron superiores en pacientes $\geq 12$ años de edad, para todas las categorías. Sin embargo, la única diferencia significativa se encontró con valores $\geq 1,0 \mathrm{mg} / \mathrm{L}$ con la vía iv ( $\mathrm{p}: 0,028$ ).

En la Tabla 4 se muestra el análisis para diferentes rangos de dosis vo y las CPs alcanzadas en cada grupo. $\mathrm{Si}$ bien un mayor porcentaje de CPs estuvo sobre los valores analizados en pacientes $\geq 12$ años en la mayoría de los casos, esta diferencia no fue significativa. En pacientes bajo 12 años, la dosis más efectiva para alcanzar CPs adecuadas para profilaxis de AI ( $\geq 0,5 \mathrm{mg} / \mathrm{L})$ fue de al

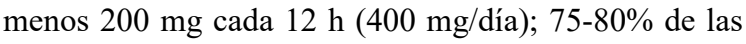
CPs alcanzaron dicho valor. En pacientes $\geq 12$ años, una dosis estándar de $400 \mathrm{mg} /$ día produjo $100 \%$ de las CPs $\geq 0,5 \mathrm{mg} / \mathrm{L}$. Sólo se encontraron $\mathrm{CPs} \geq 5,5 \mathrm{mg} / \mathrm{L}$ con dosis mayores a $400 \mathrm{mg} /$ día.

Las CPs alcanzadas con administración iv en pacientes $<12$ años $\mathrm{y} \geq 12$ años con diferentes dosis por $\mathrm{kg}$ de peso se muestran en la Tabla 5. Una mayor cantidad de CPs estuvo sobre los valores estudiados en los pacientes $\geq 12$ años respecto a los $<12$ años; no obstante, no hubo diferencias significativas. En pacientes de 12 años o más, con dosis entre 14 y $20 \mathrm{mg} / \mathrm{kg} /$ día, $80 \%$ de las CPs fueron $\geq 1 \mathrm{mg} / \mathrm{L} y \geq 2 \mathrm{mg} / \mathrm{L}$. En pacientes bajo 12 años, todas las dosis produjeron similar proporción de CPs con valores que se consideran efectivos para tratamiento de AI (50-63\% para CPs $\geq 1 \mathrm{mg} / \mathrm{L}$ y $36-40 \%$ para CPs $\geq 2 \mathrm{mg} / \mathrm{L}$ ). No hubo relación entre dosis iv y cantidad de CPs $\geq 5,5 \mathrm{mg} / \mathrm{L}$. 


\begin{tabular}{|c|c|c|c|c|c|c|}
\hline \multirow[b]{2}{*}{ Parámetro } & \multicolumn{2}{|c|}{ Dosis $<400$ mg/día } & \multicolumn{2}{|c|}{ Dosis 400 mg/día c/12 h } & \multicolumn{2}{|c|}{ Dosis $>400 \mathrm{mg} / \mathrm{dí} a \mathrm{c} / 12 \mathrm{~h}$} \\
\hline & $<12$ años & $\geq 12$ años & $<12$ años & $\geq 12$ años & $<12$ años & $\geq 12$ años \\
\hline Determinaciones de CP (n) & 11 & 10 & 12 & 8 & 5 & 3 \\
\hline $\mathrm{CP}(\mathrm{mg} / \mathrm{L})$ : mediana [rango] & $0,5[0,1-2,2]$ & $0,3[0,1-2,1]$ & $0,6[0,1-4,1]$ & $2,2[0,7-3,6]$ & $4,5[0,4-8,8]$ & $3,0[2,5-5,4]$ \\
\hline Edad (años): mediana [rango] & $5[3-11]$ & 14 [14-18] & $6,5[4-10]$ & 18 [14-18] & $6[6-9]$ & 14 \\
\hline Dosis (mg/día): mediana [rango] & $200[200-300]$ & $250[200-300]$ & 400 & 400 & $600[500-600]$ & 600 \\
\hline Dosis (mg/kg): mediana [rango] & $11,8[5,3-15,0]$ & $5,0[3,3-10,0]$ & $20,0[14,3-20,0]$ & $6,7[6,7-13,3]$ & $25,0[20,7-25]$ & $17,1[15-20]$ \\
\hline $\mathrm{CPs} \geq 0,5 \mathrm{mg} / \mathrm{L} \mathrm{n}(\%)$ & $5(45,5)$ & $4(40)$ & $9(75)$ & $8(100)$ & $4(80)$ & $3(100)$ \\
\hline CPs $\geq 1,0 \mathrm{mg} / \mathrm{L} \mathrm{n}(\%)$ & $2(18,2)$ & $2(20)$ & $5(41,7)$ & $6(75)$ & $4(80)$ & $3(100)$ \\
\hline CPs $\geq 2,0 \mathrm{mg} / \mathrm{L} \mathrm{n}(\%)$ & $1(9,1)$ & $1(10)$ & $3(25)$ & $5(62,5)$ & $3(60)$ & $3(100)$ \\
\hline CPs $\geq 5,5$ mg/L n (\%) & $0 \quad(0)$ & 0,0 & 0,0 & 0,0 & $2(40)$ & $\begin{array}{ll}0 & (0)\end{array}$ \\
\hline
\end{tabular}

\begin{tabular}{|c|c|c|c|c|c|}
\hline \multirow[b]{2}{*}{ Parámetro } & \multicolumn{2}{|c|}{ Dosis < 14 mg/kg/día } & \multicolumn{2}{|c|}{ Dosis $14-<20 \mathrm{mg} / \mathrm{kg} / \mathrm{día}$} & \multirow{2}{*}{$\begin{aligned} \text { Dosis } & \geq 20 \mathrm{mg} / \mathrm{kg} / \mathrm{día}{ }^{*} \\
& <12 \text { años }\end{aligned}$} \\
\hline & $<12$ años & $\geq 12$ años & $<12$ años & $\geq 12$ años & \\
\hline Determinaciones de CP (n) & 10 & 11 & 12 & 5 & 10 \\
\hline $\mathrm{CP}$ (mg/L): mediana [rango] & $1,1[0,22-15,5]$ & $1,6[0,8-18]$ & $1,45[0,3-2,9]$ & $2,3[0,29-6,0)$ & $1,2[0,45-4,64]$ \\
\hline Edad (años): mediana [rango] & $5,5[5-11]$ & 14 [14-18] & $6[2-10]$ & 14 [13-14] & $5[4-13]$ \\
\hline Dosis (mg/día): mediana [rango] & $360[260-600]$ & $400[320-600]$ & $350[140-500]$ & $600[400-600]$ & $400[400-600]$ \\
\hline Dosis (mg/kg): mediana [rango] & $12,8[8,1-13,9]$ & $7,1[6,0-11,9]$ & $15,8[14-19]$ & $17,1[14,6-17,1]$ & 20 [20-30] \\
\hline $\mathrm{CPs} \geq 0,5 \mathrm{mg} / \mathrm{L} \mathrm{n}(\%)$ & $7(70)$ & $11(100)$ & $9(75)$ & $4(80)$ & $10(90,9)$ \\
\hline $\mathrm{CPs} \geq 1,0 \mathrm{mg} / \mathrm{L} \mathrm{n}(\%)$ & $5(50)$ & $10(90,9)$ & $7(58,3)$ & $4(80)$ & $7(63,6)$ \\
\hline $\mathrm{CPs} \geq 2,0 \mathrm{mg} / \mathrm{L} \mathrm{n}(\%)$ & $4(40)$ & $5(45,5)$ & $5(41,7)$ & $4(80)$ & $4(36,4)$ \\
\hline CPs $\geq 5,5$ mg/L n (\%) & $3(30)$ & $2(18,2)$ & $0(0)$ & $1(20)$ & 0,00 \\
\hline
\end{tabular}

\section{Perfil de seguridad}

El resumen del tipo y frecuencia de reacciones adversas (RAM) asociadas al uso de VCZ se muestran en la Tabla 6. Ocho (30,8\%) pacientes tuvieron alguna RAM grado 3-4 en escala CTCAE. Todas fueron relacionadas al sistema hepático, mientras que $1(3,8 \%)$ los fue para el sistema ocular (fototofobia) y osteo-muscular (mialgia). De las alteraciones funcionales hepáticas y pancreáticas, las más frecuentes fueron: aumento de GGT $(23,1 \%)$, GOT $(15,4 \%)$, GPT
$(15,4 \%)$, lipasemia $(7,7 \%)$ y amilasemia $(3,8 \%)$.

De las ocho CPs $\geq 5,5 \mathrm{mg} / \mathrm{L}$ (7 pacientes), sólo cuatro se asociaron a RAM (4 pacientes), las que correspondieron a (escala CTCAE): fotofobia (2) y aumento de GGT (4); incremento de lipasemia (3); aumento de GPT (3) y mialgia (2); aumento de GOT (3) y GPT (3). De las 104 $\mathrm{CPs}<5,5 \mathrm{mg} / \mathrm{L}, 82$ fueron asociadas a alguna RAM, correspondiendo todas a hepatoxicidad. No se encontró relación entre CPs tóxicas y RAM (p: 0,5576). 


\begin{tabular}{|lcc|}
\hline $\begin{array}{l}\text { Tabla 6. Detalle de reacciones adversas asociadas a } \\
\text { voriconazol }\end{array}$ & $\mathbf{n}$ & $(\%)$ \\
\hline Parámetro & 8 & $(30,8)$ \\
\hline $\mathrm{n}$ pacientes con RAM & 22 & $(100)$ \\
$\mathrm{n}$ episodios de RAM & 20 & $(90,9)$ \\
Episodios hepatotoxicidad & 2 & $(9,1)$ \\
Otros & 8 & $(30,8)$ \\
n pacientes con hepatotoxcidad CTCAE 3-4 & 6 & $(23,1)$ \\
Pacientes con alza de GGT & 4 & $(15,4)$ \\
Pacientes con alza de GOT & 4 & $(15,4)$ \\
Pacientes con alza de GPT & 2 & $(7,7)$ \\
Pacientes con alza de lipasemia & 1 & $(3,8)$ \\
\hline Pacientes con alza de amilasemia & 1 & $(3,8)$ \\
\hline $\mathrm{n}$ pacientes con fotofobia CTAE 2 & 1 & $(3,8)$ \\
\hline $\mathrm{n}$ pacientes con mialgias CTAE 2 & & \\
\hline Algoritmo de Naranjo & 7 & $(31,8)$ \\
$\mathrm{n}$ RAM probables & 15 & $(68,2)$ \\
\hline $\mathrm{n}$ RAM posibles & & \\
\hline RAM: reacción adversa a medicamento. & & \\
\hline
\end{tabular}

\section{Discusión}

De las CPs analizadas, sólo 54 y $37 \%$ fueron $\geq 1,0$ y $2,0 \mathrm{mg} / \mathrm{L}$, respectivamente, lo que representa un bajo porcentaje. En el estudio de Soler-Palacin P y cols., realizado en población pediátrica ${ }^{24}$ sólo $50 \%$ de los pacientes tuvo $\mathrm{CPs} \geq 1,0 \mathrm{mg} / \mathrm{L}$ y en sólo $7 \%$ fueron $>5,5 \mathrm{mg} / \mathrm{L}$, muy similar a los resultados obtenidos por nosotros (54 y 7\%). En otro estudio en pacientes pediátricos, una simulación utilizando el modelo de Monte-Carlo, predijo que una dosis iv de $14 \mathrm{mg} / \mathrm{kg} /$ día, fraccionada cada $12 \mathrm{~h}$ o de 200 mg cada $12 \mathrm{~h}$ vo produciría una $\mathrm{CP}>1 \mathrm{mg} / \mathrm{L}$ en $66 \%$ de los pacientes ${ }^{17}$. Estos resultados son similares a los obtenidos en nuestro estudio, 62 y $50 \%$ de los pacientes con dosis iv de $10-14 \mathrm{mg} / \mathrm{kg} /$ día y vo $200 \mathrm{c} / 12 \mathrm{~h}$ tuvieron $\mathrm{CPs} \geq 1,0 \mathrm{mg} / \mathrm{L}$. En otro estudio efectuado por Hicks y cols $^{25}$, el porcentaje de pacientes con CPs dentro del rango 1-6 mg/L fue sólo de 51 y $36 \%$ para menores y mayores de 12 años, respectivamente. Dichos porcentajes son similares a los valores obtenidos en nuestro estudio (40,5 y $52,6 \%$ ). El mayor porcentaje de pacientes $\geq 12$ años en rango terapéutico en nuestro estudio se puede deber a que en promedio los pacientes recibieron $10,1 \pm 5,1 \mathrm{mg} / \mathrm{kg}$, mientras que el estudio de Hicks y cols., usó una dosis diaria estándar de $400 \mathrm{mg} /$ día.
Si bien no hubo diferencias significativas entre pacientes mayores y menores de 12 años en las CPs alcanzadas, este segundo grupo tuvo una tendencia a tener CPs más bajas y requirieron mayor dosis en $\mathrm{mg} / \mathrm{kg}$. Esta diferencia de dosis parece ser aún mayor en pacientes menores, situación similar a la encontrada en el estudio de SolerPalacin P y cols., (2012); los pacientes $<5$ años recibieron en promedio una dosis de $19 \mathrm{mg} / \mathrm{kg}$, fraccionado cada 12 $\mathrm{h}$, para alcanzar CPs en el rango de $1-5,5 \mathrm{mg} / \mathrm{L}^{24}$.

Debido a que no se obtuvo el dato del número de administraciones de VCZ durante el período de estudio y a la exclusión de pacientes con mediciones de CPs pico, no se obtuvo la incidencia de RAM asociadas al fármaco. Con respecto a toxicidad, $70 \%$ de las RAM registradas fueron clasificadas como "posibles", por lo que su causa puede ser multifactorial. Si bien no se encontró una relación directa con episodios de hepato-toxicidad, se registró un único episodio de fotofobia en un paciente con una CP de 15,5 mg/L. Esto concuerda con un estudio observacional en adultos donde se demostró que CPs $>5 \mathrm{mg} / \mathrm{L}$ estuvieron asociadas con una mayor incidencia de reacciones adversas neurológicas, pero no hepáticas ${ }^{9}$.

En general se obtuvieron CPs más elevadas en pacientes que recibieron el fármaco vía iv y en aquellos $\geq 12$ años, lo que puede deberse a que la vía iv evita la variabilidad de absorción que ocurre con la vo y a una metabolización más lenta en pacientes mayores.

Según nuestros resultados, la dosis más adecuada para alcanzar las CPs recomendadas para profilaxis de AI, fue de $200 \mathrm{mg}$ cada $12 \mathrm{~h}$ (400 mg/día) en todos los pacientes, de manera independiente de la edad. Para tratamiento utilizando la vo, una dosis de $600 \mathrm{mg}$ al día $(17 \mathrm{mg} / \mathrm{kg}$ ) podría ser adecuada, pero sólo se obtuvieron tres CPs en este rango.

Para tratamiento de AI en pacientes $\geq 12$ años, dosis iv entre 14 y $20 \mathrm{mg} / \mathrm{kg} /$ día, mostraron mejor comportamiento PK con $80 \%$ de las CPs $\geq 2 \mathrm{mg} / \mathrm{L}$. Será necesario corroborar estos hallazgos con una muestra de mayor tamaño, ya que contamos sólo con cinco CPs obtenidos con esta dosis (4 CPs $\geq 2 \mathrm{mg} / \mathrm{L}(80 \%)$ ). Nuestros resultados apuntan a utilizar dosis iniciales mayores a $14 \mathrm{mg} / \mathrm{kg} /$ día iv para tratamiento de AI grave, lo que ha sido recomendado por la Agencia Europea de Medicamentos (EMA) ${ }^{26}$.

Este estudio cuenta con algunas limitaciones que comentamos a continuación. Es importante mencionar que hasta $49 \%$ de la variación de las CPs de VCZ se pueden deber a polimorfismos en la enzima CYP2C $19^{27}$ y en nuestro estudio no contamos con los datos de polimorfismo de cada paciente. La dosificación individualizada de este fármaco de acuerdo a cada polimorfismo podría haber ayudado a obtener CPs terapéuticas en un plazo significativamente menor en comparación con una dosificación estándar $^{28}$. Hubo pacientes que recibieron inhibidores de la bomba de protones junto con voriconazol; en la práctica 
clínica no se ha encontrado que el uso de IBP sea una co-variante significativa para alterar el metabolismo de este fármaco ${ }^{29}$.

\section{Conclusiones}

- Es importante obtener CPs de VCZ adecuadas al inicio y durante el tratamiento y, debido a que éstas son erráticas, la monitorización farmacocinética es fundamental.

- Una dosis estándar de $200 \mathrm{mg}$ c/12 h vo, en forma independiente de la edad, parece ser adecuada para obtener CPs eficaces para profilaxis de AI. Debe recordarse que la administración oral debe realizarse una hora antes o una hora después de la administración de alimentos.

- Dosis entre $14-20 \mathrm{mg} / \mathrm{kg} /$ día iv podrían ser adecuadas para alcanzar CPs eficaces para tratamiento de AI en pacientes $\geq 12$ años.

- Los pacientes $<12$ años requerirían dosis mayores a las utilizadas en este estudio (hasta $30 \mathrm{mg} / \mathrm{kg} /$ día iv) para obtener CPs efectivas para tratamiento de AI.

- No se encontró relación entre concentraciones tóxicas y reacciones adversas.

- Es fundamental en próximos estudios realizar un análisis incluyendo además los polimorfismos de la enzima CYP2C19 para obtener una información más completa. En nuestro país, esta técnica no está disponible en la mayoría de los hospitales públicos, por lo que nuestra aproximación de dosificación parece una buena opción para alcanzar CPs adecuadas en estos pacientes.

Agradecimientos: A todo el equipo de médicos y enfermeras del hospital, en especial de las unidades de Oncología, Trasplante de Precursores Hematopoyéticos y UCI pediátrica. A todo el equipo de farmacia clínica y de farmacia hospitalizados. Al equipo de Infectología con el que trabajamos en conjunto y nos ayudamos mutuamente. En especial a José Cofré G. que nos ayudó en la revisión de este trabajo. A Carolina y Daniel del Laboratorio de Farmacocinética, con los que trabajamos en conjunto. A Brenda L. Zylbersztajn por su revisión y comentarios. A Tiffany Pardue por su apoyo y traducción al inglés.

\section{Resumen}

Introducción: Las concentraciones plasmáticas (CPs) de voriconazol (VCZ) son erráticas y en el caso de encontrarse bajo rango terapéutico para el tratamiento de aspergilosis invasora (AI) se asocian a un aumento de mortalidad. Objetivo: Analizar las CPs de VCZ obtenidas en pacientes pediátricos para alcanzar valores que se estiman efectivos para profilaxis $(\geq 0,5 \mathrm{mg} / \mathrm{L})$ y tratamiento ( $\geq 1,0$ y $2,0 \mathrm{mg} / \mathrm{L}$ ) de AI y su relación con toxicidades. Pacientes y Métodos:Análisis retrospectivo de CPs de VCZ y toxicidades asociadas obtenidas en pacientes oncológicos pediátricos desde febrero de 2013 hasta noviembre 2014. Se analizó la dosis diaria y CPs de acuerdo a la vía de administración: intravenosa (iv) u oral (vo), tipo de terapia (profilaxis y tratamiento) y edad ( $<12$ y $\geq 12$ años). Resultados: Se analizaron 112 CPs valle de 26 pacientes, con una edad promedio de 9,3 años. El 43,7\% de las CPs correspondió a administración iv. Se obtuvieron más CPs $\geq 0,5 \mathrm{mg} / \mathrm{L} \mathrm{y} \geq 1,0 \mathrm{mg} / \mathrm{L}$ con la vía iv en relación a vo $(\mathrm{p}<0,05)$. Pacientes bajo 12 años de edad recibieron mayor dosis en comparación a los $\geq 12$ años (medianas 18,6 y $9,2 \mathrm{mg} / \mathrm{kg} /$ día, respectivamente, $\mathrm{p}<0,05)$. La dosis vo más efectiva para alcanzar CPs $\geq 0,5 \mathrm{mg} / \mathrm{L}$ fue de $200 \mathrm{mg}$ cada $12 \mathrm{~h}$ en todos los pacientes (80-100\% de CPs $\geq 0,5$ $\mathrm{mg} / \mathrm{L})$. En pacientes $\geq 12$ años con dosis iv entre 14 y 20 $\mathrm{mg} / \mathrm{kg} / \mathrm{día}, 80 \%$ de las CPs fueron $\geq 1 \mathrm{mg} / \mathrm{L} \mathrm{y} \geq 2 \mathrm{mg} / \mathrm{L}$. En pacientes bajo 12 años de edad, dosis entre 8-30 mg/ $\mathrm{kg} /$ día generaron similares resultados $(50-63 \%$ para CPs $\geq 1 \mathrm{mg} / \mathrm{L}$ y $36-40 \%$ para $\mathrm{CPs} \geq 2 \mathrm{mg} / \mathrm{L}$ ). Ocho pacientes (30,8\%), tuvieron alguna reacción adversa al fármaco, no encontrándose relación con la CP alcanzada. Conclusión: Una dosis estándar vo de $200 \mathrm{mg}$ c/12 h de VCZ mostró los mejores resultados para profilaxis de $\mathrm{AI}$ en todos los pacientes. Pacientes bajo 12 años de edad requerirían dosis mayores a las utilizadas en este estudio para obtener CPs efectivas para tratamiento de AI. No se encontró relación entre CPs tóxicas y reacciones adversas.

\section{Referencias bibliográficas}

1.- Chandrasekar P H, Manavathu E. Voriconazole: a second-generation triazole. Drugs Today 2001; 37: 135-48.

2.- Herbrecht R, Denning D W, Patterson T F, Bennett J E, Greene R E, Oestmann J W, et al. Invasive Fungal Infections Group of the European Organization for Research and Treatment of Cancer and the Global Aspergillus Study Group. Voriconazole versus amphotericin
B for primary therapy of invasive aspergillosis. N Engl J Med 2002; 347: 408-15.

3.- Offner F, Cordonnier C, Ljungman P, Prentice H G, Engelhard D, De Bacquer D, et al. Impact of previous aspergillosis on the outcome of bone marrow transplantation. Clin Infect Dis 1998; 26: 1098-103.

4.- Walsh T J, Anaissie E J, Denning D W, Herbrecht R, Kontoyiannis D P, Marr K A, et al. Treatment of aspergillosis: Clinical Practice Guidelines of the Infectious Diseases
Society of America. Clin Infect Dis 2008; 46 : 327-60.

5.- Maron G M, Hayden R T, Rodríguez A, Rubnitz J E, Flynn P M, Shenep J L. Voriconazole prophylaxis in children with cancer: changing outcomes and epidemiology of fungal infections. Pediatr Infect Dis J 2013; 32 (12): e451-5.

6.- Molina J R, Serrano J, Sánchez-García J, Rodríguez-Villa A, Gómez P, Tallón D, et al. Voriconazole as primary antifungal prophylaxis 
in children undergoing allo-SCT. Bone Marrow Transplant 2012; 47 (4): 562-7.

7.- Yeh T C, Liu H C, Hou J Y, Chen K H, Huang T H, Chang C Y, et al. Severe infections in children with acute leukemia undergoing intensive chemotherapy can successfully be prevented by ciprofloxacin, voriconazole, or micafungin prophylaxis. Cancer 2014; 120 : 1255-62.

8.- Purkins L, Wood N, Kleinermans D, Greenhalgh K, Nichols D. Effect of food on the pharmacokinetics of multiple-dose oral voriconazole. Br J Clin Pharmacol 2003; 56: 17-23.

9.- Purkins L, Wood N, Ghahramani P, Greenhalgh K, Allen M J, Kleinermans D. Pharmacokinetics and safety of voriconazole following intravenous to oral dose escalation regimens. Antimicrob. Agents Chemother 2002; 46: 2546-53.

10.- Karlsson M O, Lutsar I, Milligan P A. Population pharmacokinetic analysis of voriconazole plasma concentration data from pediatric studies. Antimicrob. Agents Chemother 2009; 53 (3): 935-44.

11.- Walsh T J, Driscoll T, Milligan P A, Wood N D, Schlamm H, et al. Pharmacokinetics, safety, and tolerability of voriconazole in immunocompromised children. Antimicrob. Agents Chemother 2010; 54 (10): 4116-23.

12.- Hyland R, Jones B C, Smith D A. Identification of the cytochrome $\mathrm{P} 450$ enzymes involved in the $\mathrm{N}$ oxidation of voriconazole. Drug Metab Dispos 2003; 31 (5): 540-7.

13.- Trifilio S G, Pennick J, Pi J, Zook M, Golf K, Kaniecki, et al. Monitoring plasma voriconazole levels may be necessary to avoid subtherapeutic levels in hematopoietic stem cell transplant recipients. Cancer 2007; 109: 1532-5.

14.- Trifilio S M, Yarnold P R, Scheetz M H, Pi J,
Pennick G, Mehta J. Serial plasma voriconazole concentrations after allogeneic hematopoietic stem cell transplantation. Antimicrob. Agents Chemother 2009; 53 (5): 1793-6.

15.- Miyakis S, Van Hal S J, Ray J, Marriott D. Voriconazole concentrations and outcome of invasive fungal infections. Clin Microbiol Infect 2010; 16 (7): 927-33.

16.- Pascual A, Calandra T, Bolay S, Buclin T, Bille J, Marchetti O. Voriconazole therapeutic drug monitoring in patients with invasive mycoses improves efficacy and safety outcomes. Clin Infect Dis 2008; 46: 201-11.

17.- Neely M, Rushing T, Kovacs A, Jelliffe R, Hoffman J. Voriconazole pharmacokinetics and pharmacodynamics in children. Clin Infect Dis 2010; 50 (1): 27-36.

18.- Radford S A, Johnson E M, Warnock D W. In vitro studies of activity of voriconazole (UK-109,496), a new triazole antifungal agent, against emerging and less-common mold pathogens. Antimicrob Agents Chemother 1997; 41: 841-3.

19.- Smith J, Safdar N, Knasinski V, Simmons W, Bhavnani S M, Ambrose P G, et al. Voriconazole therapeutic drug monitoring. Antimicrob Agents Chemother 2006; 50: 1570-2.

20.- National Cancer Institute. Common Terminology Criteria for Adverse Events v4.0. NCI, NIH, DHHS. May 29, 2009. NIH publication \# 09-7473.

21.- Naranjo C A, Busto U, Sellers E M, Sandor P, Ruiz I, Roberts E A, et al. A method for estimating the probability of adverse drug reactions. Clin Pharmacol Ther 1981; 30: 239-45.

22.- Rose C. Determination of voriconazole and posaconazole in human plasma. Memphis, TN, St. Jude Children's Research Hospital. 2010.

23.- Richard Lowry, Vassar Stats: Website for
Statistical Computation (1988-2015). http:// vassarstats.net/ (consultado el 21 de noviembre de 2015).

24.- Soler-Palacin P, Frick M A, Martin-Nalda A, Lanaspa M, Pou L, Rosello E, et al. Voriconazole drug monitoring in the management of invasive fungal infection in immunocompromised children: a prospective study. J Antimicrob Chemother 2012; 67 (3): 700-6.

25.- Hicks J K, Crews K R, Flynn P, Haidar C E, Daniels C C, Yang W, et al. Voriconazole plasma concentrations in immunocompromised pediatric patients vary by CYP2C19 diplotypes. Pharmacogenomics 2014; 15 (8): 1065-78.

26.- Annex 1, Summary of product characteristics. VFEND. Disponible en http://www.ema. europa.eu/docs/en_GB/document_library/ EPAR_-_Product_Information/human/000387/ WC500049756.pdf. (consultado el 21 de octubre de 2015).

27.- Weiss J, Ten Hoevel M M, Burhenne J, Walter-Sack I, Hoffmann M M, Rengelshausen J, et al. CYP2C19 genotypes is a major factor contributing to the highly variable pharmacokinetics of voriconazole. J Clin Pharmacol 2009; 49: 196-204.

28.- Teusink A, Vinks A, Zhang K, Davies S, Fukuda T, Lane A, et al. Genotype-directed dosing leads to optimized voriconazole levels in pediatric patients receiving hematopoietic stem cell transplantation. Biol Blood Marrow Transplant 2015 Nov 23pii: S1083-8791(15)00738-7.DOI: http://dx.doi. org/10.1016/j.bbmt.2015.11.011.

29.- Dolton M J, Mikus G, Weiss J, Ray J E, McLachlan A J. Understanding variability with voriconazole using a population pharmacokinetic approach: implications for optimal dosing. J. Antimicrob. Chemother 2014; 69 (6): 1633-41. 